be due to persistence of non-acid-reflux and pepsin causing ongoing laryngeal epithelial inflammation. We investigated: (a) the prevalence of pepsin reflux in respiratory patients requiring nasendoscopy for the investigation of upper airway symptoms; (b) the performance of commonly used clinical LPR-diagnostic tools in predicting the presence of salivary pepsin.

Methods Subjects had symptoms and signs of laryngeal inflammation quantified using, the Reflux Symptom Index (RSI) and Reflux Finding Score (RFS). Salivary pepsin was measured with a lateral flow device using monoclonal antibody labelling (Peptest, RDBiomed). Patients with severe signs of laryngeal inflammation were referred for impedance-pH oesophageal studies to assess for objective evidence of reflux.

Results Of the 78 subjects recruited, $76 \%$ were female, mean age 55 (range 17-82). Ten were undergoing investigation for chronic cough, and 68 for possible vocal cord dysfunction (confirmed in 45). 30 had concomitant asthma, and 42 were prescribed anti-reflux treatment. $87 \%$ had a high RSI, and $51 \%$ a high RFS. Pepsin was detected in the saliva of $49 / 78$ subjects (63\%), and prevalence did not vary significantly between treatment group. There was a weak correlation between the RFS and pepsin concentration $(r=0.28$, $p=0.01)$ and the positive and negative predictive values for pepsin detection for those with a high RFS were $63 \%$ and $69 \%$ respectively. To date all 8 patients tested have had significant proximal reflux on impedance study, of which 6 had a positive pepsin assay.

Conclusion Salivary pepsin was frequently present in patients with upper airway symptoms, but only weakly related to clinical findings of reflux, suggesting a high prevalence of LPR that is not associated with typical laryngeal findings. The significance of such sub-clinical reflux remains to be seen however the use of pepsin assay in patients with upper airway symptoms may be most valuable in directing diagnosis in milder cases where symptoms and signs lack specificity and the condition may otherwise be missed.

\section{P152 THE IMPACT OF A SELECTIVE ORAL TRPV1 ANTAGONIST IN PATIENTS WITH CHRONIC COUGH}

doi:10.1136/thoraxjnl-2012-202678.213

IJA Smith, ${ }^{2} \mathrm{RD}$ Murdoch, ${ }^{2} \mathrm{~A}$ Newlands, ${ }^{2} \mathrm{~K}$ Smart, 'S Khalid, 'A Kelsall, ${ }^{1} \mathrm{~K}$ Holt, ${ }^{1} \mathrm{R}$ Dockry, ${ }^{1} \mathrm{~A}$ Woodcock. 'University of Manchester, Manchester, UK; ${ }^{2}$ GlaxoSmithKline, Stevenage, UK

Background Increased expression of TRPV1 receptors in the airways of chronic cough patients and heightened cough responses to inhaled capsaicin are suggestive of a role for TRPV1 receptors in chronic cough. We hypothesised that antagonism with a potent, selective, peripherally acting, oral TRPV1 antagonist, such as SB705498, would offer substantial cough symptom control.

Methods 21 patients with unexplained chronic cough (caucasian, $71 \%$ female, mean age 53yrs) participated in a double-blind, placebo-controlled, single dose, 2-period, crossover study to investigate the relationship between pharmacokinetic (PK) derived TRPV1 receptor occupancy, change in capsaicin (C5) threshold and $24 \mathrm{hr}$ cough count following $600 \mathrm{mg}$ SB705498. PK samples were taken over the dosing interval, capsaicin threshold was determined at screening, 2hrs (Cmax) and 24hrs post-dose and $24 \mathrm{hr}$ objective ambulatory cough counts were recorded on each dosing day via a cough monitor (vitaloJAK ${ }^{\mathrm{TM}}$ ) with manual counting. In addition, COLO and VAS urge to cough was measured. A battery of safety and tolerability measures were also recorded, including core body temperature.

Results TRPV1 receptor occupancy derived from plasma levels and factoring a plasma/lung ratio of 1 , was approximately $45 \%$ at $2 \mathrm{hrs}$ and $25 \%$ at $24 \mathrm{hrs}$. This translated to a 4 fold shift in the capsaicin C5 threshold at $2 \mathrm{hrs}(2 \mathrm{uM}$ to $8 \mathrm{uM})$ maintained at $24 \mathrm{hrs}$. However, there was no difference between hourly cough count profiles between placebo and SB705498 (see figure), with hourly counts of
$20-30 \mathrm{c} / \mathrm{h}$ and the characteristic nocturnal reduction. Cough counts were remarkably stable, repeated one month apart and over the 8 months of the study (including winter).

Conclusions We conclude that despite a clear relationship between receptor occupancy and engagement of the TRPV1 receptor as evidenced by the shift in the capsaicin threshold, there was no translation to any clinical efficacy parameter. This suggests peripheral TRPV1 receptor activation is not an important determinant of spontaneous cough frequency in chronic cough and that reductions in capsaicin responses do not necessarily predict anti-tussive effects.

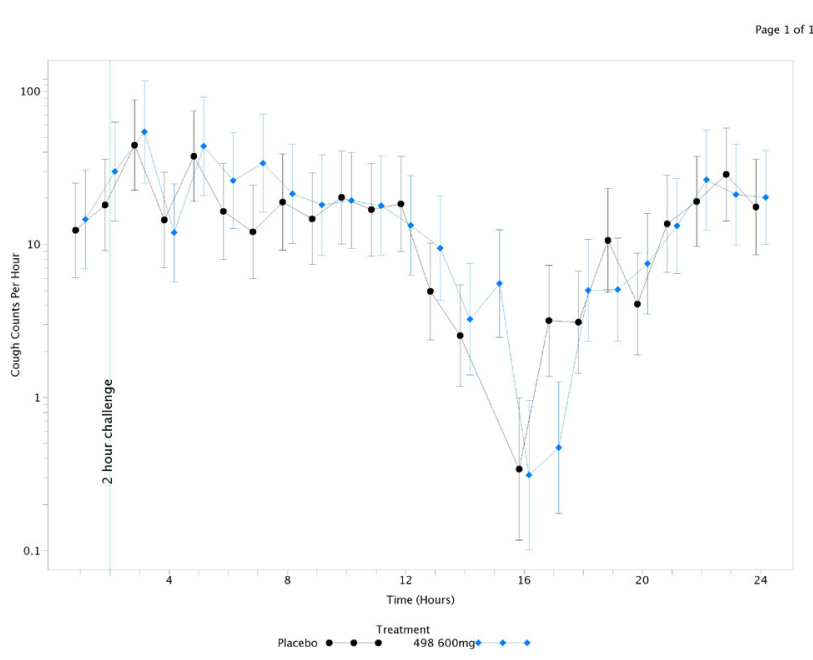

Abstract P152 Figure 1

\section{P153 THE IMPACT OF TRPV1 ANTAGONISM ON THE TREATMENT OF SEASONAL ALLERGIC RHINITIS}

doi:10.1136/thoraxjnl-2012-202678.214

${ }^{1}$ RD Murdoch, 'P Bareille, ' J Bentley, ' $\mathrm{K}$ Smart, ${ }^{2} \mathrm{~F}$ Horak. 'GlaxoSmithKline, Stevenage, UK; ${ }^{2}$ University Clinic, Vienna, Austria

Background Topical intranasal steroids are widely considered to be the most efficacious pharmacotherapy for the treatment of allergic rhinitis, and yet, for many, symptoms still remain troublesome. We hypothesise that the residual symptoms are a result of nasal neuronal hyperresponsiveness during the pollen season and should be ameliorated by a topical intranasal TRPV1 antagonist. SB705498 is a selective TRPV1 antagonist shown to produce significant inhibition in animal and human models involving nasal sensory nerves.

Methods The study involved 70, male and female, subjects with proven rhinitis in a randomised, double-blind, placebo-controlled, 3 -way incomplete block crossover design in a well validated Allergen Challenge Chamber paradigm in Vienna. Subjects received Placebo, FP (200ug), SB705498 (12mg), or FP+498. Subjects were dosed for 8 days, within the pollen season, before being exposed to a chamber challenge on the $8^{\text {th }}$ day. TNSS was the primary endpoint recorded for the 4 hours in the chamber. The comparisons of interest were FP+498 vs. FP, and 498 vs. Placebo. Additional endpoints consisted of symptoms over the 8 days of dosing, Active Anterior Rhinomanometry, Rhinoconjunctivitis OLO, PK and tolerability. Each period was separated by 14-20 days.

Results There was no evidence of a decrease in symptoms with $\mathrm{FP}+498$ compared to FP alone, or for 498 compared to placebo. Statistically significant and clinically relevant reductions in TNSS compared with Placebo were observed at all time points during the challenge for $\mathrm{FP}$ and $\mathrm{FP}+498$. The mean $(95 \% \mathrm{CI})$ reduction in weighted mean TNSS was $-2.94(-3.38,-2.50)$ for FP alone and-2.28 $(-2.79,-1.78)$ for the combination. 
There was no positive impact over FP, or 498 over Placebo, for Diary Card symptoms, ROLQ or Nasal Airflow. Tmax occurred at approximately 5 hrs post dose, in keeping with previous studies. SB705498 was very well tolerated.

Conclusions In a robust clinical model of allergic rhinitis, there was no intrinsic activity demonstrated by SB705498 and no additive effect on a background of intranasal steroids. FP was highly effective in this study. We conclude that despite engagement of the TRPV1 receptor there was no translation to clinical efficacy, suggesting redundancy in symptom pathways.

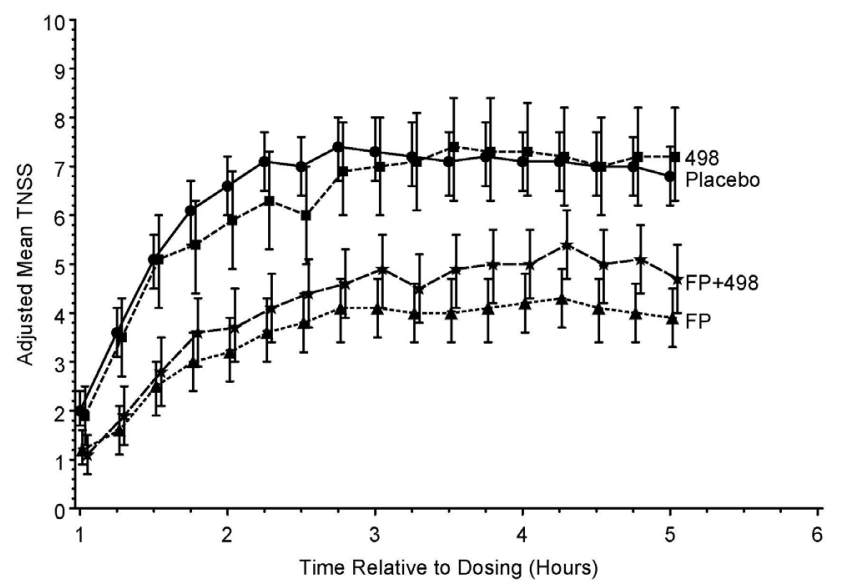

Abstract P153 Figure 1

\section{P154 TRPV1 IS NOT A TARGET FOR THE TREATMENT OF NON- ALLERGIC RHINITIS: A CLINICAL STUDY}

doi:10.1136/thoraxjnl-2012-202678.215

'RD Murdoch, 'P Bareille, 'J Bentley, 'A Newlands, ' $\mathrm{K}$ Smart, ${ }^{2} \mathrm{D}$ Patel. ' $\mathrm{G}$ laxoSmithKline, Stevenage, UK; ${ }^{2}$ Cetero Research, Mississauga, Canada

Background TRPV1 is a ligand gated ion channel activated by a range of physiological factors such at Temperature, $\mathrm{pH}$, and osmotic stress. In the nose, the TRPV1-expressing sensory c-fibres are thought to play a key role in the development of nasal hyperresponsiveness resulting in symptoms in NAR patients. We hypothesise that topical antagonism with a selective TRPV1 antagonist would offer substantial symptom control. SB705498 is a potent selective TRPV1 antagonist in animal and human models.

Methods $40 \mathrm{M} \& \mathrm{~F}$ NAR patients were enrolled into a randomised, double-blind, placebo controlled, 2 period crossover study of either $12 \mathrm{mg}$ SB705498 i.n. or placebo for 14 days with a 4 week washout. The study was conducted in a validated Environmental Challenge Chamber (Cetero) where patients were exposed to Cold Dry Air (CDA) at 14c, 15\% RH, air speed 5 feet/sec. Exposure was over the winter months in Canada. Diary card symptoms were analysed during home dosing on days when the temp was less than 14c. Two chamber sessions of $1 \mathrm{hr}$ duration, as well as medical history and neg skin prick testing, at screening were performed to establish a consistent diagnosis with a single challenge on Day $1+1 \mathrm{hr}$, Day $14+1 \mathrm{hr}$ and Day $14+24$ hr to establish the drug response. Post dose TSS (rhinorrhoea, congestion, PND), sneezing and ocular symptoms were recorded, as was acoustic rhinomanometry, ROLQ, PK and Safety monitoring.

Results The primary outcome of weighted mean TSS over the challenge period or the maximum TSS was not impacted by administration of SB705498 relative to placebo (see figure). There was no impact on sneezing, ocular symptoms, acoustic rhinimanometry, or ROLO Compared with placebo, repeated doses of SB705498 did not alleviate TSS triggered by cold in a multistimuli wild type setting.
PK analysis supported an o.d. regimen with 2 fold accumulation over the dosing period.

Conclusions In a robust clinical model of non-allergic rhinitis, intranasal SB705498 12mg o.d. for 14 days did not alleviate the symptoms of NAR triggered by the most common provocation agent: Cold Dry Air. We conclude that despite engagement of the TRPV1 receptor there was no translation to clinical efficacy, suggesting redundancy in symptom pathways.

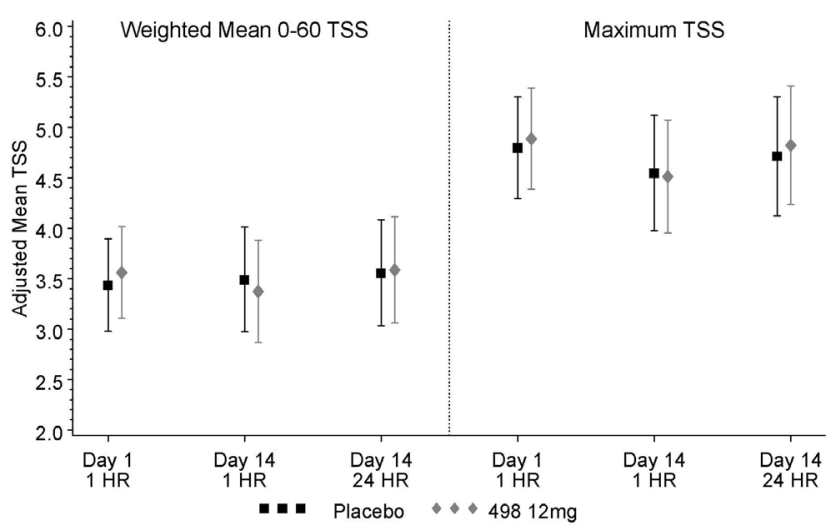

Abstract P154 Figure 1

\section{P155 VISUALISATION OF AIRWAY NERVES IN CHRONIC COUGH: TOWARDS THE IDENTIFICATION OF THE HUMAN 'COUGH RECEPTOR'}

doi:10.1136/thoraxjnl-2012-202678.216

'PW West, ${ }^{2 B J}$ Canning, 'EC Hilton, 'S Khalid, 'K Holt, 'R Abdulqawi, 'AA Woodcock, 'JA Smith. 'University of Manchester, Manchester, United Kingdom; ' $J o h n s ~ H o p k i n s$ Asthma \& Allergy Centre, Baltimore, USA

Introduction The spinal and vagal innervation of the respiratory tract is well defined, particularly in animals. These discoveries include the identification of pathways involved in provoking cough and descriptions of the guinea pig 'cough receptor'. However, many of the immunochemical features of the airway afferents described in animals have yet to be defined in humans, yet plasticity of these airway afferents may be important in the pathophysiology of chronic cough.

Objectives To define and characterise the innervation present in bronchoscopic biopsies from patients with chronic cough. We aimed to carry out the first ever whole mount immunohistochemical studies of airway nerves in cough patients, a technique that should improve visualisation of these neuronal structures and their sites of termination.

Methods Biopsy tissue was gifted from patients undergoing clinical investigation for chronic cough. Tissue, sampled from throughout the extrapulmonary airways, was immediately fixed in $4 \%$ paraformaldehyde. Non-specific antibody binding was blocked using $10 \%$ normal serum and $1 \%$ skimmed milk powder, diluted in PBS, before application of primary antibodies. Polyclonal rabbit anti-PGP9.5 (Ultraclone, UK) and monoclonal mouse anti-neurofilament (NF200, Leica Biosystems, UK) were applied at a dilution of 1:1000 and 1:200 respectively. Primary antibody binding was detected using appropriate Alexa-fluor conjugated secondary antibodies and whole mount preparations were visualised using epifluorescence and confocal microscopy. Images of biopsy staining were subject to morphometric analysis.

Results Many epithelial, subepithelial and intramuscular fibres were detected using both antibodies. Co-staining revealed that only PGP9.5 defined pulmonary neuroendocrine cells and better elucidated varicose epithelial fibres. A proportion of all fibre types were 